\title{
Characterization of TaDREB1 in wheat genotypes with different seed germination under osmotic stress
}

\author{
Meng Liu, Zeng Wang, Hong-mei Xiao ${ }^{*}$ and Yan Yang ${ }^{*}$
}

\begin{abstract}
Background: The cis-acting element DRE/CRT plays an important role in activating gene expression responsive to osmotic stress, low temperature and high-salinity. DREB1/CBF genes encode DRE-binding proteins with the function as transcript activators. TaDREB1 was also found to be induced by osmotic stress.

Methods: The dates of osmotic stress was assessed by seed germination drought resistance index; the full-length CDNA sequences of TaDREB1 gene were downloaded from NCBI datebase; identification of allelic variation and transcript expression were assessed by PCR and semi-quantitive RT-PCR analysis, respectively.

Results: Total 13 new allele variations of TaDREB1 were identified in the germplasms tested in the paper, including 5 TaDREB1-A on chromosome 3AL, 4 TaDREB1-B on chromosome 3BL and 4 TaDREB1-D on chromosome 3DL. In each variety, there existed two loci of TaDREB1-D genes, named TaDREB1-D1 and TaDREB1-D2, both of which had the similar nucleotide sequence except an 11 bp insertion in the former. In wheat seeds under osmotic stress, we did not detect the transcript expression level of TaDREB1-A and TaDREB1-B, but that of TaDREB1-D.
\end{abstract}

Conclusions: The capacity of osmotic stress tolerance was closely correlated with the expression level and tendency of TADREBT-D.

Keywords: Nucleotide polymorphism, Expression characteristics, TaDREB1, Osmotic stress resistance

\section{Background}

Drought is already widespread in many regions and one of the primary reason causing plant loss worldwide. [1]. Even in many irrigated regions, shortage of water supply allows only limited irrigation. Therefore, it is imperative to strengthen the study on osmotic stress resistance of wheat.

Five multi-gene families belong to transcript factors [bZIP (mainly AREB/ABF), DREB (AP2/EREBP), MYB/MYC, NAC and WRKY)] have been reported to be associated with drought tolerance. A number of wheat genes for transcript factors (TabZIP1, TabZIP60, TaABRE3, TaDREB1, TaPIMP1, TaNAC29 and TAWRKY44) have been shown to exhibit the induced expression during exposure to drought stress,

\footnotetext{
* Correspondence: Ihtdyx@126.com; yangyanchutao@126.com College of Life Sciences, Inner Mongolia Key Laboratory of Plant Stress Physiology and Molecular Biology, Inner Mongolia Agricultural University, Erdos Road, Hohhot 010018, Inner Mongolia, China
}

suggesting that these genes may be used for improving stress tolerance in wheat [2-9].

Dehydration-responsive element binding (DREB) proteins constitute a large family of transcription factors regulating some functional genes closely related to drought, high-salinity and low temperature $[10,11]$. Arabidopsis genome has six DREB1/CBF genes, namely $D R E B 1 A / C B F 3, \quad D R E B 1 B / C B F 1, \quad D R E B 1 C / C B F 2$, $D R E B 1 D / C B F 4, \quad D D F 1 / D R E B 1 F$ and DDF2/DREB1E [12]. DREB genes feature three conserved regions, an EREBP/AP2 DNA binding domain $[13,14]$, an $\mathrm{N}$-terminal nuclear localization signal, and a conserved Ser/Thr-rich region adjacent to the EREBP/AP2 domain $[15,16]$. The characteristics of above three regions determine the DREB characteristics [17]. Most of the functional studies on $D R E B$ transcription factors were focused on Arabidopsis thaliana before 2002, and then in wheat, rice, soybean, cotton and other plants [18-24]. $D R E B$ factors have been extensively characterized and

(C) The Author(s). 2018 Open Access This article is distributed under the terms of the Creative Commons Attribution 4.0 International License (http://creativecommons.org/licenses/by/4.0/), which permits unrestricted use, distribution, and reproduction in any medium, provided you give appropriate credit to the original author(s) and the source, provide a link to the Creative Commons license, and indicate if changes were made. The Creative Commons Public Domain Dedication waiver (http://creativecommons.org/publicdomain/zero/1.0/) applies to the data made available in this article, unless otherwise stated. 
grouped into several molecular classes based on similarities in the function or the amino acid sequence of the proteins they encode [24]. DREB1 and DREB2 are two main subgroups of the DREB subfamily, involved in two different signal transduction pathways under cold and dehydration, respectively [25]. DREB1 genes include DREB1-A, DREB1-B, DREB1-C,DREB1-D,DREB1-E and $D R E B 1 F$ [26]. TaDREB1 genes were located on chromosomes 3A, 3B and 3D [11], and isolated from a drought-induced cDNA library of wheat, which was found to be induced by low temperature, abscisic acid (ABA), salinity and drought [27]. The cDNA length of TaDREB1 is $1292 \mathrm{bp}$, including $837 \mathrm{bp}$ open reading frame, 251 bp 5'-UTR and 204 bp 3'-UTR [18]. The study results showed that the heterogeneity of TaDREB1 gene haplotypes is inconsistent in drought-resistant materials or in drought-sensitive wheat materials, indicating the complexity of drought resistance. In addition, the nucleic acid polymorphisms of TaDREB1 gene are richer in wheat [26].

The present research aimed at identifying the seed germination resistance varieties under osmotic stress for wheat breeding program, searching the new allelic variation of TaDREB1, looking for SNPs, InDels and transcript expression level associated with seed germination tolerance under osmotic stress, and charactering the transcript expression level of TaDREB1-A, TaDREB1-B and TaDREB1-D in wheat varieties or lines with different values of GDRI (germination drought resistance index).

\section{Methods}

\section{Plant materials}

Six spring wheat genotypes were used for cloning of TaDREB1-A, TaDREB1-B and TaDREB1-D genes, including three osmotic stress-resistant genotypes (081783, 08-1826 and 07-6239 with relative germination rate values of $55.98,47.74$ and $47.11 \%$, respectively) and three osmotic stress-susceptible ones (08dongzhong 275, Zhangye 1 and $05 \mathrm{~cm} 178$ with relative germination rate values of 1.27, 0.51 and $0 \%$, respectively). Eighty Chinese spring wheat genotypes, with different osmotic stress tolerance from China Spring-sown Wheat Region (CSWR) were used for identifying the osmotic stress tolerance (Table 1). Mature seeds of $08-1783$ and Zhangye 1 were used to extract RNA for analysis of mRNA transcription.

\section{Methods}

\section{Assay for seed germination drought tolerance}

Osmotic stress was assessed based on seed germination drought resistance index (GDRI). Ears were harvested at dough-yellow ripening stage, hand-threshed and sterilized with $\mathrm{HgCl}_{2}$, and then placed in plastic petri dishes on filter paper with $6 \mathrm{~mL}$ of treatment solution $(-1.00 \mathrm{MPa})$ at room temperature, and distilled water was used as a control. Germinated seeds were counted and removed daily. The number of germinated seeds was investigated on the 2nd, 4th, 6th and 8th days (seeds with over $1 \mathrm{~mm}$ length of radicle were counted). The GDRI values was calculated according to the following formula:

$$
\begin{aligned}
& \text { GDRI } i=\frac{G I s i}{\text { GIci }} \times 100 \% \\
& G D R I=\sum_{i=1}^{3} \text { GDRI } i / 3 \\
& G I=(1.00 \mathrm{nd} 2+0.75 \mathrm{nd} 4+0.50 \mathrm{nd} 6+0.25 \mathrm{nd} 8) / N \times 100
\end{aligned}
$$

GDRI-germination drought resistance index of seeds per reparation.

GIs-germination index of seeds under stress.

GIc-germination index of seeds as control.

nd2, nd4, nd6, nd8 -represents the number of seeds germinated on day $2,4,6$ and 8 , respectively.

$\mathrm{N}$-represents the total number of seeds.

Then using the membership function method in fuzzy mathematic, the average germination index of wheat under osmotic stress was calculated to evaluate the difference of osmotic stress resistance among the varieties $[28,29]$.

$$
\begin{aligned}
& \mathrm{U}\left(\mathrm{x}_{\mathrm{j}}\right)=\frac{\mathrm{xj}-\mathrm{xjmin}}{\mathrm{xjmax}-\mathrm{xjminj}}=1,2,3 \\
& \mathrm{U}\left(\mathrm{x}_{\mathbf{j}}\right)=1-\frac{\mathrm{xj}-\mathrm{xjmin}}{\mathrm{xjmax}-\mathrm{xjmin} j}=1,2,3 \\
& \mathrm{D}=\sum_{j=1}^{n}\left[\mathrm{U}(\mathrm{xj}) \frac{|\mathrm{rj}|}{\sum_{j=1}^{n}|\mathrm{rj}|}\right]=1,2,3
\end{aligned}
$$

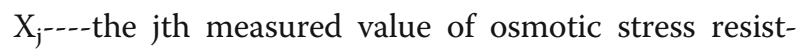
ance index;

$\mathrm{X}_{\mathrm{j}}$ max-----the maximum value of the $\mathrm{jth}$ osmotic stress resistance index.

$\mathrm{X}_{\mathrm{j}}$ min-----the minimum value of the $\mathrm{jth}$ osmotic stress resistance index.

$\mathrm{U}\left(\mathrm{x}_{\mathrm{j}}\right)$--the membership function value of the $\mathrm{jth}$ index.

Rj-----The correlation coefficient between the jth index and the comprehensive 106 osmotic stress resistance coefficient. 
Table 1 Primer sequence used in this study

\begin{tabular}{|c|c|c|c|}
\hline Primer & Primer sequence $\left(5^{\prime}-3^{\prime}\right)$ & Fragment size (bp) & Anneal temp. $\left({ }^{\circ} \mathrm{C}\right)$ \\
\hline DREB1-A1F & CGGAACCACTCCCTCCATCTC & 1107 & 62 \\
\hline DREB1-A1R & CGGTTGCCCCATTAGACGTCA & & \\
\hline DREB1-A2F & CTGGCACCTCCATTGCTGAC & 599 & 63 \\
\hline DREB1-A2R & AGTACATGAACTCAACGCACAGGACAAC & & \\
\hline DREB1-BF & CCCAACCCAAGTGATAATAATCT & 716 & 58 \\
\hline DREB1-BR & TTGTGCTCCTCATGGGTACTT & & \\
\hline DREB1-D1F & TCGTCCCTCTTCTCGCTCCAT & 1190 & 63 \\
\hline DREB1-D1R & GCGGTTGCCCCATTAGACATCG & & \\
\hline DREB1-D2F & CTGGCACCTCCATTGCCGAT & 596 & 64 \\
\hline DREB1-D2R & AGTACATGAACTCAACGCACAGGACAAC & & \\
\hline DREB $U$ & TCGTCCCTCTTCTCGCTCCATGG & 493 & 66 \\
\hline DREB D & GGGCATGGCG CCGCATGG & & \\
\hline DREB1-AF & ATGAACAGGAAGAAGAAAGTGCGC & 593 & 62 \\
\hline DREB1-AR & TTCTCAAATCATTGCTCACT TCTITC & & \\
\hline DREB1-BF & ATGACCAGGAAGAAGAAAGTGCGC & 585 & 60 \\
\hline DREB1-BR & TCATTGCTCACTTCTITITTCACCTTAT & & \\
\hline DREB1-DF & ATGAACAGGAAGAAGAAAGTGCGC & 455 & 62 \\
\hline DREB1-DR & TCCTTCCCATCAGAAGGATGTGAC & & \\
\hline$\beta$-actin $F$ & GTTCCTGGAATTGCTGATCGCAT & 410 & 65 \\
\hline$\beta$-actin R & CATTATTTCATACAGCAGGCAAGC & & \\
\hline
\end{tabular}

$R_{j} / \sum n j=1 r j-.---$ As the indicator weight, indicating the importance of the jth index in all indicators.

D---The evaluated value of each osmotic stress resistance indicator for each species under mannitol stress.

If the measured index is positively correlated with the osmotic stress resistance of the genotypes, then formula (1) is used to calculate the membership function value, otherwise (2). Using D values to evaluate the osmotic stress resistance of the species, that is, $D>0.80$ is for Level 1 (strong resistance), $0.50<\mathrm{D}<0.80$ is for Level 2 (resistance), $0.30<\mathrm{D}<0.5$ is for Level 3 (weak resistance), $\mathrm{D}<0.30$ is for Level 4 (sensitive). The entire osmotic stress resistance index was treated with Excel system.

\section{Primer design}

Based on the full-length cDNA sequence of TaDREB1 gene(AF303376), the primers were designed using DNAMAN software and were synthesized in Takara Biotechnology (Dalian) Co. Ltd. in China (Table 2).

\section{PCR amplification and semi-quantitive RT-PCR analysis}

For each varieties, $3 \sim 5$ seeds were selected and cultured in a clean culture dish at $25^{\circ} \mathrm{C}$ for $5 \sim 7 \mathrm{~d} .1 \sim 2 \mathrm{~g}$ leaves were frozen by liquid nitrogen. Then genomic
DNA was extracted by method of CTAB. The TaDREB1-A, TaDREB1-B and TaDREB1-D were amplified by specific primers (Table 1 ) in genotypes with different osmotic stress tolerance.

PCR reaction was performed in a PTC-100 TMProgrammable Thermal Controller in a total volume of $15 \mu \mathrm{L}$, including $30 \mathrm{ng}$ genomic DNA, $10 \times$ PCR reaction Mix buffer $1.5 \mu \mathrm{L}, 0.3 \mu \mathrm{M}$ of each primer, $0.5 \mathrm{U}$ of Taq DNA polymerase (TaKaRaCo., China). It was carried out using the following programs: initial denaturation at $94{ }^{\circ} \mathrm{C}$ for $5 \mathrm{~min}$; 35 cycles of $72{ }^{\circ} \mathrm{C}$ for 30-90s, annealing at $58{ }^{\circ} \mathrm{C} \sim 64{ }^{\circ} \mathrm{C}$ for $30 \sim 90$ s, $72{ }^{\circ} \mathrm{C}$ for $30 \sim 90$ s and a final extension at $72{ }^{\circ} \mathrm{C}$ for $10 \mathrm{~min}$. The PCR products recovered by agarose gel were cloned into the cloning vector PMD19-T (TaKaRaCo., China). The sequencing was performed by Nanjing Kingsley Sequencing Co., Ltd. Sequencing data analysis was performed using DNAMAN software (https://www.lynnon.com/pc/framepc.html).

Total RNA was extracted from the mature dry seeds treated with - 1.00 MPa mannitol for $0 \mathrm{~h}, 12 \mathrm{~h}, 24 \mathrm{~h}$ and 36 h, using TaKaRa Mini BEST plant RNA extraction kit (TaKaRa Co., China). The concentration and purity of the total RNA extracted was determined using a NanoDropND-2000C spectrophotometer. According to the manuscript, cDNA was synthesized with the reverse transcriptase kit RTaseM-MLV (TaKaRa Co., China). RT-PCR reaction was performed in a PTC-100 
Table 2 Values of GDRI in 80 spring wheat varieties and lines

\begin{tabular}{|c|c|c|c|c|c|}
\hline $\mathrm{No}$ & Varieties and lines & GDRI (2015)\% & GDRI (2016)\% & Average GDRI (\%) & D values \\
\hline 1 & 08-1783 & 22.57 & 89.39 & 55.98 & 1.00 \\
\hline 2 & $07-5866$ & 22.77 & 74.18 & 48.47 & 0.87 \\
\hline 3 & $08-1826$ & 29.71 & 65.78 & 47.74 & 0.85 \\
\hline 4 & $07-6239$ & 21.66 & 72.57 & 47.11 & 0.84 \\
\hline 5 & Bayou 2 & 22.83 & 68.27 & 45.55 & 0.81 \\
\hline 6 & $34-206$ & 6.74 & 83.69 & 45.22 & 0.81 \\
\hline 7 & Linyou 2 & 22.95 & 66.17 & 44.56 & 0.80 \\
\hline 8 & 08-1718 & 9.16 & 77.47 & 43.31 & 0.77 \\
\hline 9 & 08-2294 & 9.51 & 75.42 & 42.46 & 0.76 \\
\hline 10 & 08-3348 & 7.8 & 76.92 & 42.36 & 0.76 \\
\hline 11 & Jinsui0095 & 1.66 & 80.11 & 40.88 & 0.73 \\
\hline 12 & Qingchun37 & 6.07 & 75.46 & 40.77 & 0.73 \\
\hline 13 & Bayou1 & 7.38 & 73.62 & 40.5 & 0.72 \\
\hline 14 & Linyou 1 & 10.99 & 68.57 & 39.78 & 0.71 \\
\hline 15 & 73B609 & 13.47 & 63.03 & 38.25 & 0.68 \\
\hline 16 & $8916-40$ & 12.94 & 63.41 & 38.17 & 0.68 \\
\hline 17 & Bi 1ama0 & 12.39 & 62.47 & 37.43 & 0.67 \\
\hline 18 & Wu E32-1 & 13.01 & 61.71 & 37.36 & 0.67 \\
\hline 19 & Yong2739 & 0 & 70.26 & 35.13 & 0.63 \\
\hline 20 & Jiu9996 & 2.08 & 63.58 & 32.83 & 0.59 \\
\hline 21 & Jinsui8145 & 7.57 & 50.14 & 28.86 & 0.52 \\
\hline 22 & 08dongzhong2455 & 7.26 & 48.33 & 27.8 & 0.50 \\
\hline 23 & Qingchun556 & 12.07 & 43.37 & 27.72 & 0.50 \\
\hline 24 & $05 \mathrm{~cm} 220$ & 0.75 & 53.45 & 27.1 & 0.48 \\
\hline 25 & 05-1173 & 3.92 & 46.72 & 25.32 & 0.45 \\
\hline 26 & Lafan8 & 8.97 & 39.75 & 24.36 & 0.44 \\
\hline 27 & $95 \mathrm{y} 16$ & 11.41 & 32.34 & 21.88 & 0.39 \\
\hline 28 & Zhongning31051 & 8.62 & 32.69 & 20.65 & 0.37 \\
\hline 29 & Ganchun20 & 11.54 & 26.67 & 19.1 & 0.34 \\
\hline 30 & Ningchun10 & 4.35 & 32.09 & 18.22 & 0.33 \\
\hline 31 & Bafeng1 & 16.78 & 18.43 & 17.6 & 0.31 \\
\hline 32 & NingPS184 & 1.01 & 33.76 & 17.39 & 0.31 \\
\hline 33 & La2676-9 & 1.92 & 32.84 & 17.38 & 0.31 \\
\hline 34 & Yemao & 16.34 & 18.18 & 17.26 & 0.31 \\
\hline 35 & G47 & 16.92 & 15.74 & 16.33 & 0.29 \\
\hline 36 & Zhongguochun & 9.29 & 22.43 & 15.86 & 0.28 \\
\hline 37 & C1845 & 5.73 & 24.5 & 15.12 & 0.27 \\
\hline 38 & 08dongzhong6741 & 4.55 & 25.12 & 14.83 & 0.26 \\
\hline 39 & Jiaoyuan356 & 16.47 & 10.92 & 13.7 & 0.24 \\
\hline 40 & Xiaobing32 & 4.12 & 22.59 & 13.35 & 0.24 \\
\hline 41 & 08-1699 & 0.64 & 25.93 & 13.28 & 0.24 \\
\hline 42 & 08dongzhong1791 & 11.54 & 14.22 & 12.88 & 0.23 \\
\hline 43 & $07-6228$ & 3.03 & 22.19 & 12.61 & 0.23 \\
\hline 44 & Ganchun22 & 8 & 16.77 & 12.39 & 0.22 \\
\hline
\end{tabular}


Table 2 Values of GDRI in 80 spring wheat varieties and lines (Continued)

\begin{tabular}{|c|c|c|c|c|c|}
\hline No & Varieties and lines & GDRI (2015)\% & GDRI (2016)\% & Average GDRI (\%) & $\mathrm{D}$ values \\
\hline 45 & Bafeng3 & 3.17 & 19.93 & 11.55 & 0.21 \\
\hline 46 & $03 \mathrm{~cm}-338$ & 0 & 22.46 & 11.23 & 0.20 \\
\hline 47 & Qinghai 932 & 0 & 22.37 & 11.18 & 0.20 \\
\hline 48 & 08dongzhong1807 & 1.76 & 18.31 & 10.03 & 0.18 \\
\hline 49 & $05-5371$ & 0.52 & 17.03 & 8.77 & 0.16 \\
\hline 50 & Luobupin & 16.19 & 0.88 & 8.54 & 0.15 \\
\hline 51 & Wu M27-2 & 0 & 16.67 & 8.33 & 0.15 \\
\hline 52 & YK6-325 & 1.45 & 15.15 & 8.3 & 0.15 \\
\hline 53 & 08-353 & 6.32 & 9.89 & 8.11 & 0.14 \\
\hline 54 & Bafeng6 & 8.58 & 7.37 & 7.98 & 0.14 \\
\hline 55 & Gelanni & 1.96 & 13.91 & 7.93 & 0.14 \\
\hline 56 & $82,170-1$ & 8.7 & 6.67 & 7.68 & 0.14 \\
\hline 57 & Wu M437 & 3.57 & 11.76 & 7.67 & 0.14 \\
\hline 58 & Yong 2352 & 6.11 & 8.78 & 7.45 & 0.13 \\
\hline 59 & 08dongzhong2417 & 8.8 & 5.28 & 7.04 & 0.13 \\
\hline 60 & 08dongzhong3008 & 10.71 & 2.42 & 6.56 & 0.12 \\
\hline 61 & Ningzi08A751 & 0 & 12.79 & 6.39 & 0.11 \\
\hline 62 & 08dongzhong550 & 3.21 & 8.75 & 5.98 & 0.11 \\
\hline 63 & y31 & 2.26 & 9.67 & 5.96 & 0.11 \\
\hline 64 & Moyin45 & 4.76 & 7.03 & 5.9 & 0.11 \\
\hline 65 & 08dongzhong1146 & 7.69 & 3.96 & 5.83 & 0.10 \\
\hline 66 & Lanyou5074 & 0.39 & 8.67 & 4.53 & 0.08 \\
\hline 67 & $08-3410$ & 1.37 & 6.25 & 3.81 & 0.07 \\
\hline 68 & $08 \mathrm{H} 415$ & 3.23 & 4.38 & 3.8 & 0.07 \\
\hline 69 & $07-6751$ & 2.3 & 4.76 & 3.53 & 0.06 \\
\hline 70 & Hetao3 & 0 & 6.97 & 3.48 & 0.06 \\
\hline 71 & Zhangchun11 & 0 & 6.25 & 3.13 & 0.06 \\
\hline 72 & Yong 2356 & 0 & 5.69 & 2.84 & 0.05 \\
\hline 73 & $08 \mathrm{H} 274$ & 0.34 & 3.56 & 1.95 & 0.03 \\
\hline 74 & Jiusandasui7788 & 2.63 & 1.23 & 1.93 & 0.03 \\
\hline 75 & Shentai1 & 0.96 & 2.2 & 1.58 & 0.03 \\
\hline 76 & 08dongzhong275 & 0 & 2.53 & 1.27 & 0.02 \\
\hline 77 & 08dongzhong1809 & 0 & 2.5 & 1.25 & 0.02 \\
\hline 78 & Ningchun35 & 2.33 & 0 & 1.16 & 0.02 \\
\hline 79 & Zhangye1 & 1.02 & 0 & 0.51 & 0.01 \\
\hline 80 & $05 \mathrm{~cm} 178$ & 0 & 0 & 0 & 0.00 \\
\hline
\end{tabular}

TMProgrammable Thermal Controller with a total volume of $15 \mu \mathrm{L}$, including $30 \mathrm{ng}$ template cDNA, $1.5 \mu \mathrm{L} 10 \times \mathrm{PCR}$ reaction mix buffer, $0.3 \mu \mathrm{M}$ of each primer, $0.5 \mathrm{U}$ of Taq DNA polymerase(TaKaRaCo., China). PCR amplification were $94{ }^{\circ} \mathrm{C}$ for $5 \mathrm{~min}$; 35 cycles of $72{ }^{\circ} \mathrm{C}$ for 30 s, annealing at $62{ }^{\circ} \mathrm{C}$ for $30 \mathrm{~s}$, $72{ }^{\circ} \mathrm{C}$ for 30 s and a final extension at $72{ }^{\circ} \mathrm{C}$ for $10 \mathrm{~min}$. The PCR products were detected by $1 \%$ agarose gel.

\section{Results}

Identification of GDRI in $\mathbf{8 0}$ varieties and lines

The osmotic stress resistance during germination period of wheat seeds was tested by seed germination drought resistance index (GDRI). The GDRI values of the 80 genotypes showed consistence over the 2 years $(R=0.467$, $P=0.000)$, with mean values and standard deviations were $6.99 \pm 6.82$ and $30.47 \pm 26.84$ in the year of 2015 and 2016, respectively (Table 1, Fig. 1). 


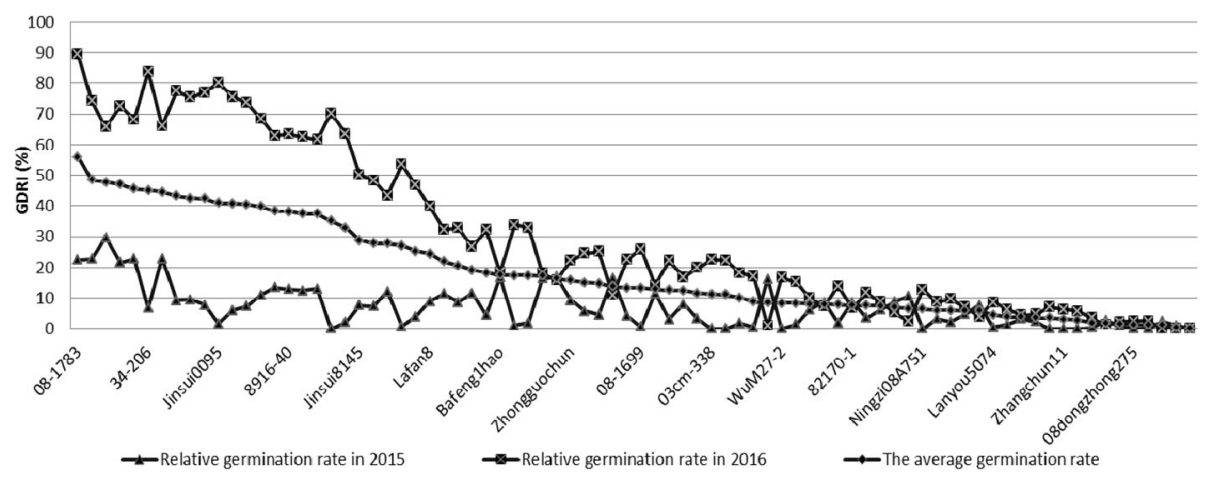

Fig. 1 The trend of the seed germination osmotic stress index in 80 spring wheat varieties and lines

The degree of osmotic stress resistance during seed germination period was evaluated by the $\mathrm{D}$ values of membership function method in fuzzy mathematics. The analysis of results showed that seven genotypes (08-1783, 07-5866, 08-1826, 07-6239, Bayou 2, 34206 and Linyou 2) belong to the level 1 osmotic stress tolerance $(0.8<\mathrm{D}<1)$, accounting for $8.75 \%$; Sixteen belong to the level 2 osmotic stress tolerance $(0.5<\mathrm{D}<0.8)$, accounting for $20.00 \%$; Fifteen belong to the level 3 osmotic stress tolerance $(0.5<\mathrm{D}<0.3)$, according for 18.75\%; And another 46 ones belong to the level 4 osmotic stress tolerance (sensitive, $0<\mathrm{D}<0.3$ ), according for $57.50 \%$ (Fig. 2).

\section{Identification of allelic variation of TaDREB1 gene}

The specific primers of DREB1-A1, DREB1-A2, DREB1-B, $D R E B 1-D 1$ and $D R E B 1-D 2$ were used to amplify the TaDREB1-A1, TaDREB1-B1 and TaDREB1-D1, respectively, in six genotypes (including three osmotic stress-resistant materials and three osmotic stress-sensitive ones). Total 13 new allele variations of TaDREB1-A1, TaDREB1-B1 and TaDREB1-D1 were found and named according

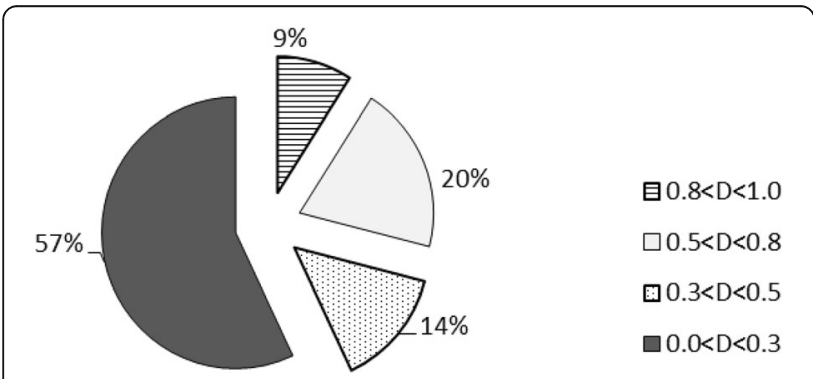

Fig. 2 Distribution pattern of seed germination osmotic stress resistance of 80 spring wheat varieties and lines. Note: $D=\sum_{j=1}^{n}$ $\left[U(x j) \frac{|r j|}{\sum_{j=1}^{\prime}|r j|}\right] j=1,2,3(3)$, D value means the evaluated value of each drought resistance indicator for each species under mannitol stress; $0.8<D<1.0$ is strong resistance, $0.8>D>0.5$ is resistance, $0.5>D>0.3$ is weak resistance, $0.3>D>0$ is sensitive. to the 2005 Supplement of the Wheat Gene Catalogue [30] in the study, i.e. TaDREB1-A11, TaDREB1-A12, TaDREB1-A13, TaDREB1-A14 and TaDREB1-A15 on chromosome 2AL; TaDREB1-B11, TaDREB1-B12, TaDREB1-B13 and TaDREB1-B14 on chromosome 2BL; TaDREB1-D11, TaDREB1-D12, TaDREB1-D21 and TaDREB1-D22 on chromosome 2DL.

Compared with TaDREB1-A (GenBank accession number: DQ195070.1), 38 SNPs were found in the full sequence of TaDREB1-A1, the frequency of SNPs being 2.27\%. Among these, 4 SNPs were observed in 5'UTR, 7 SNPs located in the first exons, 23 SNPs found in introns, and the other SNPs were found in the second exons of all alleles of TaDREB1-A1 except TaDREB1-A14. The mutation in 939 bp site is non-synonymous from hydrophobic weak glycine (G) to hydrophobic strong aspartic acid (D), while the mutations in other 3 sites are synonymous. Three base deletions were detected, among which 1 single base $\mathrm{T}$ is missed at position $40 \mathrm{bp}$ in 5'UTR of all TaDREB1-A1 variations, and other 2 bp TT deletions occurred at position $453 \mathrm{bp}$, in introns of all TaDREB1-A1 variations except TaDREB1-A14. 3 insertions were found at position $540 \mathrm{bp}(\mathrm{T}), 608 \mathrm{bp}$ (CTG), 629 bp (CT) in introns of all TaDREB1-A1 variations except TaDREB1-A14 (Additional file 1: Figure S1).

33SNPs were found in the full sequence of TaDREB1-B1, the frequency of SNPs was $4.78 \%$. Among them, 6 SNPs were found in 5'UTR, twenty-seven SNPs were located in exons of TaDREB1-B11 and TaDREB1-B12. One $\mathrm{T}$ is inserted at $485 \mathrm{bp}$ site of coding region. T-A, E-D, G-R, T-I, A-P, K-N, E-R, V-I, S-E, N-Q changes were found in the protein sequences. Most of the amino acid mutations were detected in TaDREB1-A13 genotypes, and the hydrophilicity is enhanced after the amino acid mutation. Lysine (k) is missed. The changes were not consistent with the osmotic stress resistance of the wheat genotypes (Additional file 2: Figure S2).

Two genes of TaDREB1-D existed simultaneously on D chromosome of all genotypes, named TaDREB1-D1 amplified with the primer set DREB1-D1F/R and 
TaDREB1-D2 amplified with the primer set DREB1-D2F/R. TaDREB1-D1 and TaDREB1-D2 had the similar nucleotide sequence except a $11 \mathrm{bp}$ insertion in the former. In addition, two alleles of TaDREB1-D1 were identified, named TaDREB1-D11 and TaDREB1-D12, and also two alleles of TaDREB1-D2 were identified, named TaDREB1-D21 and TaDREB1-D22. TaDREB1-D11 had the same sequence with TaDREB1-D (GenBank accession number: DQ195068.1), while eight SNPs were detected in TaDREB1-D12, of which 6 SNPs presented at position 1205 bp (G-A), 1264 bp (C-G), 1282 bp (A-G), $1340 \mathrm{bp}$ (A-G), $1429 \mathrm{bp}(\mathrm{C}-\mathrm{A})$ and $1490 \mathrm{bp}$ (A-G), respectively. Among the SNPs, three mutations changed the amino acids from A (1.8) to A (4.4), $\mathrm{H}(-3.2)$ to $\mathrm{R}(-4.5)$ and $A(1.8)$ to $E(-3.5)$, respectively. The hydrophilicity of amino acids changed was generally enhanced. Another 2 SNPs were detected in 3'UTR of TaDREB1-D12. Moreover, TaDREB1-D21 had the same sequence with TaDREB1-D11 (DQ195068.1) except a 11 bp deletion (CCCATGCGGCG) at position of $481 \mathrm{bp}-491 \mathrm{bp}$ in intron; And TaDREB1-D22 had the same sequence with TaDREB1-D12 except a 11 bp deletion (CCCATGCGG CG) at position of $481 \mathrm{bp}-491 \mathrm{bp}$ in intron and a $3 \mathrm{bp}$ (ATT) insertion at positon 1645-1647 bp in 3 'UTR (Additional file 3: Figure S3). However, several SNPs were found between TaDREB1-D21 and TaDREB1-D22, the same as between TaDREB1-D11 and TaDREB1-D12.

\section{Expression characterization of TaDREB1-A, TaDREB1-B, and TaDREB1-D in two genotypes with different seed germination osmotic stress tolerances}

In order to define the expression patterns of the three TaDREB1 homologues and their relationship with osmotic stress tolerance, semi-quantitative RT-PCR analysis was carried out to determine the expression levels of TaDREB1-A, TaDREB1-B, and TaDREB1-D in two wheat genotypes differing in seed germination osmotic stress tolerance, using the ACTIN gene as an internal control. The results showed that the transcriptions of TaDREB1-A and TaDREB1- $B$ genes were not detected in dry seeds and seeds treated with $-1.00 \mathrm{MPa}$ mannitol for 12,24 and $36 \mathrm{~h}$ via agarose gel electrophoresis. However, the expression level of TaDREB1-D had the tendency to increase gradually and then decrease when treated with $-1.00 \mathrm{MPa}$ mannitol for 0 h, 12 h, $24 \mathrm{~h}$ and $36 \mathrm{~h}$. Higher transcript expression level was detected in seeds treated by mannitol than in dry seeds. The highest transcript expression level came from osmotic stress-resistant line $08-1783$ for $24 \mathrm{~h}$ treatment and from osmotic stress-sensitive variety Zhangye 1 after $12 \mathrm{~h}$ treatment (Fig. 3).

\section{Discussion}

osmotic stress is the most significant environmental stress in agriculture worldwide, so the improvement of

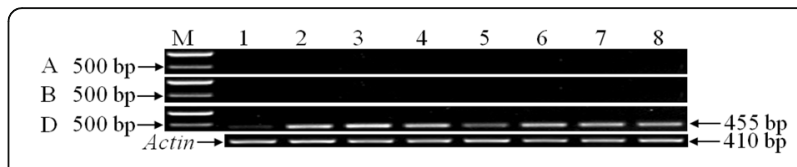

Fig. 3 Expression of TaDREB1 Gene in Different osmotic stress Resistance and Different osmotic stress-treated Wheat Seeds by RT-PCR.M: DL-2000 DNA marker; Lanes 1-4: lines of 08-1783 $(G D R I \%=55.98)$ (dry seeds treated with $-1.00 \mathrm{MPa}$ mannitol for $0 \mathrm{~h}$, $12 \mathrm{~h}, 24 \mathrm{~h}$ and $36 \mathrm{~h})$; Lanes 5-8:variety of Zhangye $1(\mathrm{GDR} / \%=0.51))$ (dry seeds treated with - 1.00 MPa mannitol for $0 \mathrm{~h}, 12 \mathrm{~h}, 24 \mathrm{~h}$ and $36 \mathrm{~h}$ ).

grain yield under water limitation is increasingly targeted in plant breeding program. Seed germination osmotic stress resistance is important for wheat cultivation, especially in seeding stage. TaDREB play an important role in response to drought stress [2]. Compared with the result studied by Wei et al. [11], much rich allelic variations were founded in our study. From the 6 genotypes tested, a total of 13 gene variations (TaDREB1-A11, TaDREB1-A12, TaDREB1-A13, TaDREB1 -A14, TaDREB1-A15, TaDREB1-B11, TaDREB1-B12, TaDREB1-B13, TaDREB1-B14, TaDREB1-D11, TaDREB1D12, TaDREB1-D21 and TaDREB1-D22) were identified, indicating that much richer allelic TaDREB1variations existed in common wheat. However, no relationship was found between the SNPs and GDRI for the time being.

In addition, two TaDREB1-D genes was detected in each of the 6 wheat genotypes tested, named TaDREB1-D1 and TaDREB1-D2; And two alleles of TaDREB1-D1 (TaDREB1-D11 and TaDREB1-D12) and two alleles of TaDREB1-D2 (TaDREB1-D21 and TaDREB1-D22) were identified. It was interesting to find that TaDREB1-D21 had the same sequence with TaDREB1-D11 (DQ195068.1) except a 11 bp deletion (CCCATGCGGCG) at position of 481 bp-491 bp in intron; And TaDREB1-D22 had the same sequence with TaDREB1-D12 except a 11 bp deletion (CCCATGCGGCG) at position of $481 \mathrm{bp}-$ $491 \mathrm{bp}$ in intron and a $3 \mathrm{bp}$ (ATT) insertion at positon 1645-1647 bp in 3 'UTR (Additional file 3: Figure S3). However, several SNPs were found between TaDREB1-D21 and TaDREB1-D22, the same as between TaDREB1-D11 and TaDREB1-D12. The base similarity between TaDREB1-D11 and TaDREB1-D21 (or between TaDREB1-D12 and TaDREB1-D22) was higher than between the two alleles of TaDREB1-D1 (or between the two alleles of TaDREB1-D2). Besides, TaDREB1-D11 and TaDREB1-D21 always appeared together in the same genotype, so as for TaDREB1-D12 and TaDREB1-D22. The results indicated that the duplication of TaDREB1-D gene occurred after the SNP mutations, and the copy of TaDREB1-D11 and TaDREB1-D12 (TaDREB1-D21 and TaDREB1-D22) was independent in different genotypes or environments. 
Higher transcript expression level of TaDREB1-D presented in seeds treated with mannitol, but no transcript product detected in TaDREB1-A and TaDREB1-B, the results showed that TaDREB1-D was more important to seed germination under osmotic stress than TaDREB1-A and TaDREB1-B. There were some reports that some genes homologies to DREB1 were weakly induced by osmotic stress $[10,19,31]$, so same resulit were founded in TaDREB1- $A$ and TaDREB1-B because of no transcript product detected in agrose gel. In addition, according to the complicated function of DREB in Arabidopsis thaliana, rice, soybean, cotton and other plants [18-23] Bohnert et al. 1995 and the transcript expression character of TaDREB1-D, more work needs to clarify the function of TaDREB1-D in further experiment.

When seeds were treated with $-1.00 \mathrm{MPa}$ mannitol, the time of the highest transcript expression level of TaDREB1-D presented at $24 \mathrm{~h}$ in the line with osmotic stress resistance, but the highest transcript expression level of TaDREB1-D presented at $12 \mathrm{~h}$ in the variety with osmotic stress sensitivity. If the time that the highest transcript expression level of TaDREB1-D appeare in more genotypes with different osmotic stress tolerance and relate with seed germonation tolerance under osmotic stress, it could be used as a potential marker to identify the genotypes with more seed germination drought resistance in breeding program, and then to breed the stable and high yield varieties in dry farming conditions. It is necessary to characterize genetic resources based on osmotic stress adaptation, determine suitable genotypes, and use them to improve the wheat resistance. In the germplasms tested, 7 genotypes belong to level 1 osmotic stress tolerance were identified, (08-1783, 07-5866, 08-1826, 07-6239, Bayou 2, 34-206 and Linyou 2), which can be used as the parents in wheat breeding program for seed germination osmotic stress resistance.

\section{Conclusions}

In summy, our study was intended to identify the seed germination resistance varieties, search the new allelic variation of TaDREB1, and character the transcript expression level of TaDREB1-A, TaDREB1-B and TaDREB1-D in wheat varieties or lines with different values of GDRI.

The results indicated that seven genotypes belong to the level 1 osmotic stress tolerance, which might be used in wheat breeding for drought resistence; thirteen new allele variations of TaDREB1 were identified in the germplasms tested, in addition, TaDREB1-D1 and TaDREB1-D2 existed in each variety; In wheat seeds under osmotic stress, no transcript expression level of TaDREB1-A and TaDREB1-B were detected, but that of TaDREB1-D, and the capacity of osmotic stress tolerance was closely correlated with the expression level and tendency of TaDREB1-D.

\section{Additional files}

Additional file 1: Figure S1. Sequence comparison of the TaDREB1-A11, TaDREB1-A12,TaDREB1-A13,TaDREB1-A14,TaDREB1-A15 and TaDREB1-A (DQ195070 .1), SNPs shown with boldface letters. (DOCX $40 \mathrm{~kb}$ )

Additional file 2: Figure S2. Sequence comparison of the TaDREB1-B11 TaDREB1-B12,TaDREB1-B13,TaDREB1-B14 and TaDREB1-B (DQ195069 .1), SNPs shown with boldface letters. (DOCX $19 \mathrm{~kb}$ )

Additional file 3: Figure S3. Sequence comparison of the TaDREB1-D11, TaDREB1-D12,TaDREB1-D21,TaDREB1-D22 and TaDREB1-D (DQ195068.1) insertions are shadowed, SNPs shown with boldface letters. (DOCX $23 \mathrm{~kb}$ )

\section{Abbreviations}

$A B F$ : ABRE binding factor; $A R E B$ : $A B A$ responsive element binding protein; CBF: C-repeat binding factor; $C R T$ : $C$ repeat;

CTAB: Hexadecyltrimethylammonium bromide; DREB: Dehydration-responsive element binding; GDRI: Germination drought resistance index;

UTR: Untranslated regions

\section{Funding}

The work was supported by grants from the Excellent Young Scientist Foundation of Inner Mongolia Agricultural University of China (2014XYQ-18) and Natural Science Foundation of China (31760382).

Availability of data and materials

The authors declare that the data supporting the findings of this study are available within the article.

Authors' contributions

$M L$ and $Z W$ performed the experiments and wrote the paper. $H X$ and $Y Y$ designed the experiment and assisted in writing the paper. All authors read and approved the final manuscript.

Ethics approval and consent to participate

Not applicable.

Consent for publication

Not applicable.

\section{Competing interests}

The authors declare that they have no competing interests.

\section{Publisher's Note}

Springer Nature remains neutral with regard to jurisdictional claims in published maps and institutional affiliations.

Received: 26 March 2018 Accepted: 25 July 2018

Published online: 01 August 2018

\section{References}

1. Vinocur B, Altman A. Recent advances in engineering plant tolerance to abiotic stress: achievements and limitations. Curr.opin.biotechnol. 2005:16:123

2. Egawa C, Kobayashi F, Ishibashi M, Nakamura T, Nakamura C, Takumi S. Differential regulation of transcript accumulation and alternative splicing of a dreb2 homolog under abiotic stress conditions in common wheat. Genes Genet Sys. 2006:81:77.

3. Cao XY, Chen M, Zhao S, XU CYF, Li LC, Yu YH, Liu YN, Ma YZ. Isolation and functional analysis of the bzip transcription factor gene taabp1 from a chinese wheat landrace. J Integr Agric. 2012;11:1580-91.

4. Zhang L, Zhao G, Xia C, Jia J, Liu X, Kong X. A wheat r2r3-myb gene, tamyb30-b, improves drought stress tolerance in transgenic arabidopsis. J Exp Bot. 2012;63:5873-85

5. Zhang ZY, Liu X, Wang XD, Zhou MP, Zhou XY, Ye XG, Wei XN. An r2r3 myb transcription factor in wheat, tapimp1, mediates host resistance to bipolarissorokiniana and drought stresses through regulation of defenseand stress-related genes. New Phytol. 2012;196:1155. 
6. Zhang L, Zhang L, Xia C, Zhao G, Liu J, Jia J, Kang X. A novel wheat bzip transcription factor, tabzip60, confers multiple abiotic stress tolerances in transgenic arabidopsis. Physiol Plantarum. 2015;153(4):538.

7. Huang QJ, Wang Y, Li B, Chang JL, Chen MJ, Li KX, Yang GX, He GY. Tanac29, a nac transcription factor from wheat, enhances salt and drought tolerance in transgenic Arabidopsis. BMC Plant Biol. 2015;15:268.

8. Wang $X$, Zeng J, Li Y, Rong $X$, Sun J, Sun T, Li M, Wang L, Feng Y, Chai R, Chen M, Chang J, Li K, Yang G, He G. Expression of tawrky44, a wheat wrky gene, in transgenic tobacco confers multiple abiotic stress tolerances. Front Plant Sci. 2015;6:615

9. Wang J, Li Q, Mao X, Li A, Jing R. Wheat transcription factor taareb3 participates in drought and freezing tolerances in Arabidopsis. Inter J Bio Sci. 2016;12:257.

10. Liu Q, Kasuga M, Sakuma Y, Abe H, Miura S, Yamaguchishinozaki K, Shinozaki K. Two transcription factors, dreb1 and dreb2, with an erebp/ap2 dna binding domain separate two cellular signal transduction pathways in drought- and low-temperature-responsive gene expression, respectively, in arabidopsis. Plant Cell. 1998;10:1391-406.

11. Wei B, Jing R, Wang C, Chen J, Mao X, Chang X. Dreb1, genes in wheat (triticum aestivum. L): development of functional markers and gene mapping based on SNPs. Mol Breeding. 2009;23:13-22.

12. Sakuma Y, Liu Q, Dubouzet JG, Abe H, Shinozaki K, Yamaguchishinozaki K. Dna-binding specificity of the erf/ap2 domain of arabidopsis, drebs, transcription factors involved in dehydration-and cold-inducible gene expression. Biochem Bioph Res Co. 2002;290:998-1009.

13. Knight H, Veale EL, Warren GJ, Knight MR. The sfr6 mutation in arabidopsis suppresses low-temperature induction of genes dependent on the crt/dre sequence motif. Plant Cell. 1999;11:875.

14. Lee H, Xiong L, Gong Z, Ishitani M, Stevenson B, Zhu JK. The arabidopsis hos 1 gene negatively regulates cold signal transduction and encodes a ring finger protein that displays cold-regulated nucleo-cytoplasmic partitioning. Genes Dev. 2001;15:912.

15. Jofuku KD, Montagu MV, Okamuro JK. Control of arabidopsis flower and seed development by the homeotic gene apetala2. Plant Cell. 1994;6:1211

16. Ohmetakagi $M$, Shinshi $H$. Ethylene-inducible dna binding proteins that interact with an ethylene-responsive element. Plant Cell. 1995:7:173-82.

17. Kanaya E, Nakajima N, Morikawa K, Okada K, Shimura Y. Chracterization of the transcriptional activator CBF1 from Arabidopsis thaliana. Evidence for cold denaturation in regions outside of the DNA binding domain. Bio Chem. 1999;274:16068-76.

18. Shen YG, Zhang WK, He SJ, Zhang JS, Liu Q, Chen SY. An erebp/ap2-type protein in triticum aestivum was a dre-binding transcription factor induced by cold, dehydration and aba stress. Theor Appl Genet. 2003;106:923-30.

19. Kidokoro S, Watanabe K, Ohori T, Moriwaki T, Maruyama K, Mizoi J. Soybean dreb1/cbf-type transcription factors function in heat and drought as well as cold stress-responsive gene expression. Plant J. 2015:81505-18.

20. Navarro M, Marque G, Ayax C, Keller G, Borges JP, Marque C, Teulières C. Complementary regulation of four eucalyptus cbf genes under various cold conditions. J Exp Bot. 2009:60:2713.

21. Ito Y, Katsura K, Maruyama K, Taji T, Kobayashi M, Seki M, Shinozaki K, Yamaguchi-Shinozaki K. Functional analysis of rice dreb1/cbf-type transcription factors involved in cold-responsive gene expression in transgenic rice. Plant Cell Physiol. 2006:47:141.

22. Qin F, Sakuma Y, Li J, Liu Q, Li YQ, Shinozaki K, Yamaguchi-Shinozaki K. Cloning and functional analysis of a novel dreb1/cbf transcription factor involved in cold-responsive gene expression in zea mays I. Plant Cell Physiol. 2004;45:1042.

23. Dubouzet JG, Sakuma Y, Ito Y, Kasuga M, Dubouzet EG, Miura S, Seki M, Shinozaki K, Yamaguchi-Shinozaki K. OsDREB genes in rice, Oryza sativa L., encode transcription activators that function in drought-, high-salt- and cold-responsive gene expression. Plant J. 2003;33:751-63.

24. Bohnert HJ, Nelson DE, Jensen RG. Adaptations to environmental stresses. Plant Cell. 1995;7:1099-111.

25. Stockinger EJ, Gilmour SJ, Thomashow MF. Arabidopsis thaliana cbf1 encodes an ap2 domain-containing transcriptional activator that binds to the c-repeat/dre, a cis-acting DNA regulatory element that stimulates transcription in response to low temperature and water deficit. P Natl Acad Sci USA. 1997:94:1035-40.

26. Yousfi S, Márquez AJ, Betti M, Araus JL, Serret MD. Gene expression and physiological responses to salinity and water stress of contrasting durum wheat genotypes. Chinese J Plant Eco. 2016;58:48.
27. Kurahashi Y, Terashima A, Takumi S. Variation in dehydration tolerance, ABA sensitivity and related gene expression patterns in D-genome progenitor and synthetic hexaploid wheat lines. Int J Mol Sci. 2009;10:2733-51.

28. Yang JW, Zhu JG, Wang SG, Sun DZ, Shii YG, Chen WG. Drought-resistance of local wheat varieties in Shanxi province of China: a comprehensive evaluation by using GGE biplot and subordinate function. Chinese J Appl Ecol. 2013;24:1031.

29. Brookes AJ. The essence of SNPs. Gene. 1999;234:177-86.

30. Mcintosh RA, Dubcovsky J, Rogers WJ, Morris CF, Appels R, Xia XC. Catalogue of gene symbols for wheat: 2005 supplement. Proc intern wheat Genet symp paestum Italy. 2001;57:27-37.

31. Magome H, Yamaguchi S, Hanada A, Kamiya Y, Oda K. Dwarf and delayedflowering 1, a novel arabidopsis mutant deficient in gibberellin biosynthesis because of overexpression of a putative ap2 transcription factor. Plant $J$. 2004;37:720-9.

\section{Ready to submit your research? Choose BMC and benefit from:}

- fast, convenient online submission

- thorough peer review by experienced researchers in your field

- rapid publication on acceptance

- support for research data, including large and complex data types

- gold Open Access which fosters wider collaboration and increased citations

- maximum visibility for your research: over $100 \mathrm{M}$ website views per year

At BMC, research is always in progress.

Learn more biomedcentral.com/submissions 